\title{
Virtual Environments and Cognitive Tests for Dementia Diagnosis
}

\author{
Juan Manuel Fernández Montenegro and Vasileios Argyriou \\ Faculty of Science, Engineering and Computing \\ Kingston University \\ Kingston upon Thames, UK \\ J.Fernandezmontenegro@kingston.ac.uk,Vasileios.Argyriou@kingston.ac.uk
}

\begin{abstract}
Dementia is a syndrome characterised by the decline of mental skills such as memory, reasoning, language or perceptual interpretation. High rate of deaths and high cost for detection, treatments and patient's care count amongst its consequences. The aim of this work is the creation of low cost and efficient tools to help with the detection of Alzheimer. In addition, current game technologies have proved to be a convenient tool for healthcare due to the flexibility to create certain situations provided by Virtual Environments (VEs). Therefore, our objective is the creation of novel e-health applications, accessible to all patients, using new affordable technologies combined with Human Computer Interaction (HCI) systems and VEs.
\end{abstract}

Keywords: Alzheimer's disease; Dementia; Early detection; Screening tests; HCI; Virtual environments

\section{INTRODUCTION}

Dementia is a syndrome characterised by the decline of mental skills such as memory, reasoning, language or perceptual interpretation. Alzheimer is the most common type; one of the most noticeable symptoms is the difficulty in learning new information. In addition, when the disease advances, there are other symptoms such as disorientation, mood and behaviour changes; confusion about events, dates and places; suspicions about family, friends and caregivers; and difficulty speaking, writing and walking (Alzheimer's Association, 2016). Dementia is characterized by the decline of mental abilities (Whalley \& Breitner, 2009) and the expected lifetime is five years in average (Alzheimer's Research UK, 2012). According to the World Health Organization $1.43 \%$ of the estimated deaths around the world in 2030 will be caused by Alzheimer's disease or other dementias. One in three people that indirectly die as a result of dementia will be men and the remaining will be women (more women are affected as a result of a gender average lifespan). Dementia is irreversible and it deteriorate over time. Therefore, it is important to detect dementia at very early stages in order to reduce the deterioration speed. Furthermore, early detection is essential in order to allow the patient to obtain more benefits from the treatment and to inform them and their families beforehand about future difficulties. The worldwide cost of dementia estimated in 2010 was US\$604 billion (Wimo \& Prince, 2010) and it was estimated to increase $85 \%$ by 2030 . Therefore, it is essential to develop affordable diagnosis and support tools to limit the increasing cost of dementia. One of the proposed initiatives is focused on the implementation of e-health solutions to reduce cost and to make the health systems universally accessible (Lewis, Synowiec, Lagomarsino \& Schweitzer, 2012). 
When it comes to detecting Alzheimer, different types of invasive and non-invasive tests are available. Invasive methods require obtaining data within the patient's body. These tests are not always safe and comfortable for the patient and some of them are unbearably painful, such as blood extraction or lumbar puncture (Alzheimer's Association, 2016; Han, Gruhl, Beckett, Dodge, Stricker, Farias \& Mungas, 2012; Kadmiri , 2015). On the other hand, non-invasive tests are harmless and despite the fact that some of them require the use of external devices for a certain period, these methods are more user friendly during the diagnosis process (López-deIpiña, Alonso, Barroso, Faundez-Zanuy, Ecay, Solé-Casals, Travieso, Estanga \& Ezeiza, 2012). Additionally, most of the non-invasive Alzheimer techniques can be applied at any location, such as at home without the supervision of technology or medical experts.

The most common non-invasive Alzheimer tests for diagnosis are cognitive tests. A well-known problem of these tests is their lack of adaptability according to the IQ of the patient, since most of the tasks that integrate an Alzheimer's detection cognitive test usually are too simple to evaluate high IQ patients. However, the use of computerised tests helps to create intelligence adaptable cognitive tests (Wild, Howieson, Webbe, Seelye \& Kaye, 2008). Moreover, based on the available technology, it is possible to design new types of tests that are more effective using for example virtual environments (VEs). Virtual environments provide additional advantages to cognitive tests, since it is possible to immerse the patient in a controlled situation, (Taekman \& Shelley, 2010; Tarnanas, Schlee, Tsolaki , Müri, Mosimann \& Nef, 2013; Weibel \& Wissmath, 2011).

In this paper, we propose novel cognitive and executive function based, non-invasive screening tests for early Alzheimer's diagnosis implemented as an e-health tool. The remainder of this paper is organised as follows: section 2 describes previous related work on Alzheimer's diagnosis. Section 3 describes a proposed methodology, details on the evaluation process and the obtained results; and in section 4, the conclusions.

\section{PREVIOUS WORK}

In this section, we discuss related work on Alzheimer. Early stage detection has significant importance in reducing the progress speed (Abe, Toya \& Inoue, 2013). The main types of tests can be separated to cognitive and non-cognitive ones. Cognitive tests encompass methods that evaluate patients' cognition. Non-cognitive tests are regarded as all other methods, invasive or non-invasive, used to detect and diagnose dementia.

\subsection{Non-cognitive tests}

Regarding the non-cognitive approaches, in (Alzheimer's Association, 2016; Han, Gruhl, Beckett, Dodge, Stricker, Farias \& Mungas, 2012) some of the methods used to detect dementia are based on defining potential biomarkers. These biomarkers have been proved an accurate indicator of the presence of Alzheimer (Alzheimer's Association, 2016). Nevertheless, since obtaining these biomarkers require invasive techniques, these tests are usually painful. In addition, they are not validated yet in large groups as an accurate and reliable tool for Alzheimer's disease detection so they cannot be used in medical clinics (Alzheimer's Association, 2016). 
Other non-cognitive techniques presented in (Unay \& Ekin, 2011; Akgul \& Ekin, 2010) that provide accurate results are based on Magnetic Resonance Imaging (MRI). These techniques compare the head MRI data of the patient with the corresponding data of patients with Alzheimer. Nevertheless, since acquisition of MR images involves the use of medical equipment that is not easily accessible and the process is unpleasant (claustrophobic and noisy), these methods are not suitable for testing large groups of people due to cost and time, neither mental disease patients' for safety and discomfort reasons.

Abe, Toya \& Inoue (2013) present another detection method using sensors in patients' homes to identify certain events. This non-cognitive approach is non-invasive and it requires only the consent of the patient to install the sensors. The main issue with these methods is that the obtained results are not precise enough (less than $75 \%$ detection rates) to provide an accurate technique for dementia diagnosis at early stages. Another non-cognitive method proposed by Aztiria , Ugarte \& Izaguirre (2013) uses a sensor on the patient's foot in order to analyse their gait (step length and step height) since it reflects patients' dementia level. However, the gait measurement method still has some drawbacks such as the patient has to wear a device for long periods and the results obtained, despite being promising, are still not useful since they have not been tested in Alzheimer patients.

\subsection{Cognitive tests}

The most common tests for early detection are the cognitive ones. These tests are using problem solving tasks and questions, in order to detect a cognitive impairment. The Mini Mental State Examination (MMSE) is one of the most well-known and used tests (Fountoulakis, Tsolaki, Chantzi \& Kazis, 2000). The results provided by this test demonstrate high accuracy and specificity. Nevertheless, the results show a ceiling effect, since the test is not complex enough for high IQ patients (Fountoulakis, Tsolaki, Chantzi \& Kazis, 2000). Saint Louis University Mental Status (SLUMS) examination is another screening tool used to detect mild dementia similar to MMSE (Cruz-Oliver, Malmstrom, Roegner, Tumosa \& Grossberg, 2014). There are other cognitive tests focused mainly on a single cognitive task. For example, the memorization of interacting objects in the Visual Association Test (VAT) (Lindeboom, Schmand, Tulner, Walstra \& Jonker, 2002) or the detection of the prevalence of the right ear when sounds are memorized by Alzheimer's patients during the Dichotic Listening test (Duchek \& Balota, 2005; Oppy \& Dowe, 2011).

Doctors also perform physical examinations, such as eye movement or reflexes, as part of the patient's mental state evaluation (Alzheimer's Association, 2016) since visuospatial functions and visual processes go into decline due to Alzheimer (Quental, Brucki, \& Bueno, 2013; Pereira, Marina von Zuben, Aprahamian \& Forlenza, 2014). Therefore, some of the visual methods to evaluate the mental state of a patient include the measure of the reaction time to certain stimulus, the assessment of attention or the evaluation of patient's visual memory (Pereira, Marina von Zuben, Aprahamian \& Forlenza, 2014; Crutcher, Calhoun-Haney, Manzanares, Lah, Levey \& Zola, 2009).

As it has been proved in (Quental, Brucki, \& Bueno, 2013); Alzheimer patients have some visual deficits such as visual attention deficits or visual memory problems. These impairments can be 
detected using eye tracking devices that measure eye movements such as fixations and saccades. For example, Pereira, et al. (Pereira, Marina von Zuben, Aprahamian \& Forlenza, 2014) analysed different methods that use eye movement to determine visual impairments in Alzheimer patients. These methods compare eye movements of healthy people and Alzheimer patients such as the fixation duration, refixations or saccade orientation. As a result, most of the studies revealed an increment of saccades, defects in fixations and slow pursuit movements.

Recently, due to new advances in technology, Virtual Environments (VEs) have started to form part of medical tests and rehabilitation therapies (Cho, Ku, Cho, Kim, Kang, Jang \& Kim, 2014). The work presented by Tarnanas, Tsolaki, Nef, Müri \& Mosimann (2014) demonstrates that through the use of VEs it is possible to improve the results from previous cognitive tests as it reduces the floor and the ceiling effects by creating tests that adapt to the patients' IQ (Wild, Howieson, Webbe, Seelye \& Kaye, 2008); and it is possible to increase the immersion of the patient in the task that is in progress. Tarnanas, Schlee, Tsolaki, Müri, Mosimann \& Nef (2013) work requires large screens to display the environment and some depth sensors to recognise the patients' gestures. Therefore it is not portable and cost efficient, making it unsuitable for e-health applications.

This paper is an extension of the work in (Fernández \& Argyriou, 2015) providing a novel noninvasive Alzheimer's detection screening test based on VEs is proposed and advances on the previous ones are suggested to allow adaptability. The use of new immersive technologies such as virtual reality glasses provides a huge improvement in immersing the subjects in a VE. The proposed tool is the first Alzheimer detection test that provides full immersion using Virtual Environments. In addition, it allows the patients to focus more on their tasks. Also, it is possible to adapt some of the tasks to the patient's IQ level during the test, avoiding the ceiling effect. The tests are based on the examination of cognitive abilities and they try to provide a user friendly and comfortable environment for the patients. Furthermore, the proposed approach do not consider only memory tasks, but introduce novel approaches based on the concept of differentiating between reality and a virtual world and a new cognitive test that aims to separate normal and abnormal events or objects.

\section{PROPOSED METHODOLOGY}

Novel, low cost, non-invasive, cognitive Alzheimer screening tests based on VEs have been developed. VR glasses are the devices used to increase the immersion, whereas a depth camera is used to track the patients' movements and animate their avatars, see Figure 1. Since, Alzheimer's patients are mainly elder people; all tasks are performed seated on a chair requiring only minimal movements. Virtual reality glasses and depth sensor tools are affordable and can be reused by other patients, resulting in a low cost Alzheimer's detection application.

The main scene was designed such as to recreate the doctor's office or any other real room that the patient can recognise (office, living room), see Figure 2. This room is a dynamic space with self-motion objects. It is also possible to interact with the room through the keyboard or with the virtual glasses. The tests were then defined taking advantage of the virtual environment versatility, such as the absence of physical limits. Regarding the real environment, the patient is sitting in a comfortable chair about two meters in front of the depth sensor and he/she is helped to put on the Virtual Reality glasses, see Figure 3. Once the patient feels comfortable with the 
glasses, we proceed with the tests. The test supervisor has to be in front of the computer locally or remotely in order to control the software application and to make notes of the results (Figure 4).

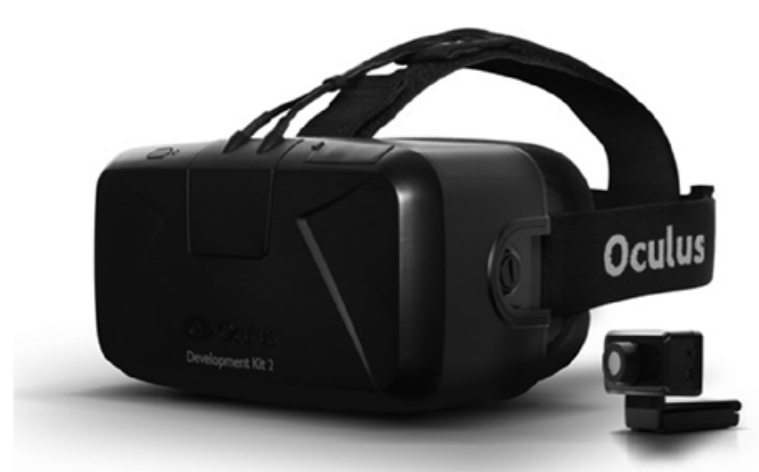

Oculus Rift DK2

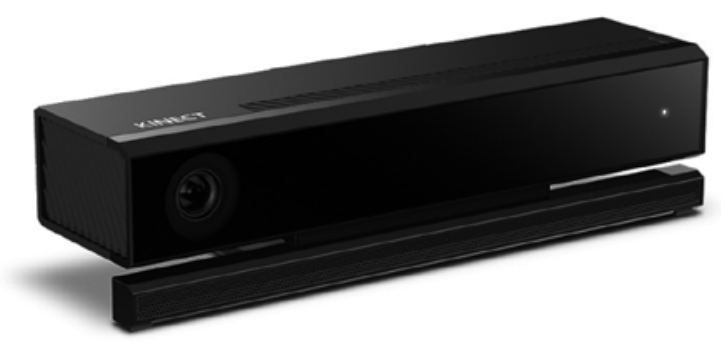

Kinect Sensor

Figure 1. Virtual Reality glasses (Oculus Rift DK2) and Depth sensor (Microsoft Kinect 2 sensor) are the devices required to perform the tests.

Although most of the tests are automatic and the patient only has to follow the instructions, some actions, such as starting a new task or playing sounds requires the interaction of the doctor/instructor. This is to provide enough time for the patient to perform the actual tasks or to add a degree of randomness and adaptability.
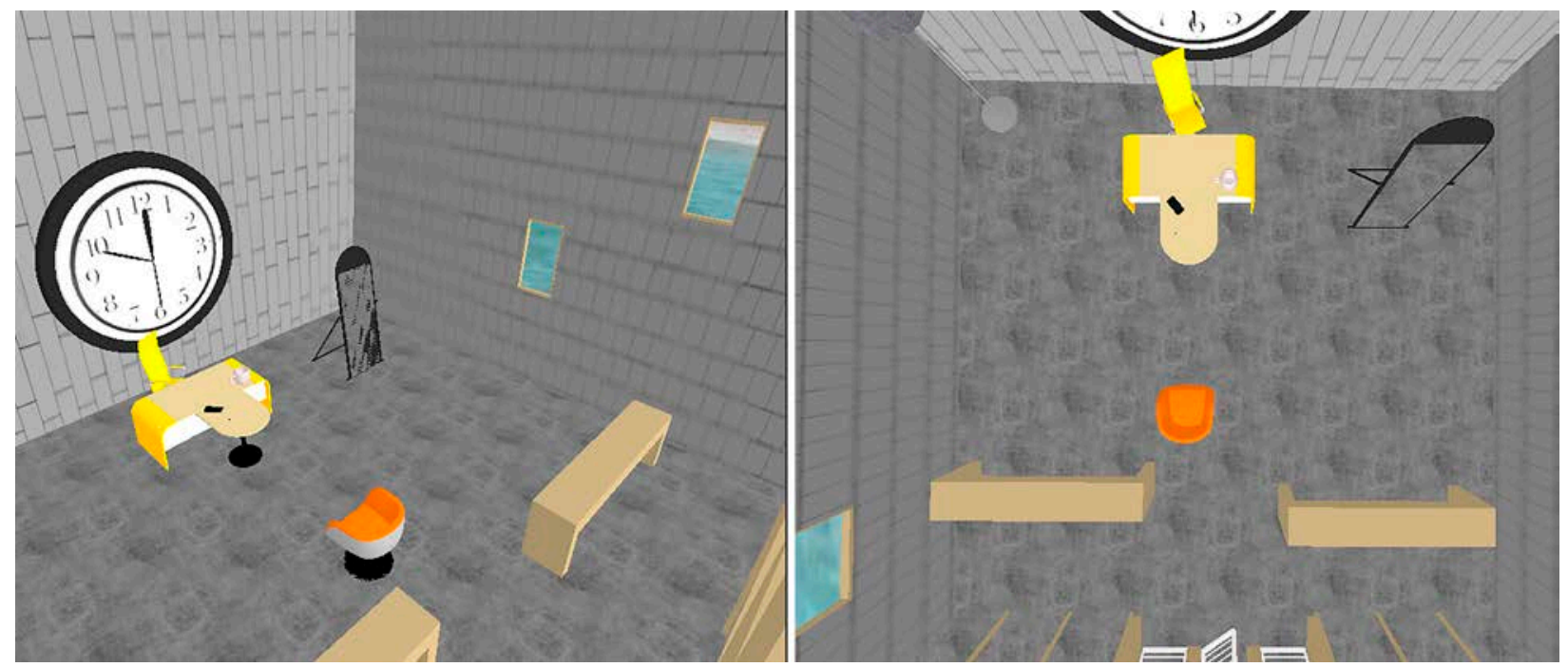

Figure 2. Virtual Reality Room: Top down and Corner view.

All the proposed tests should be performed under the supervision of a healthy person following the instructions on the screen or the test's information sheets. It is recommended that the 
supervisor during the tests is a doctor either locality or remotely, since additional information may be obtained during the whole process.

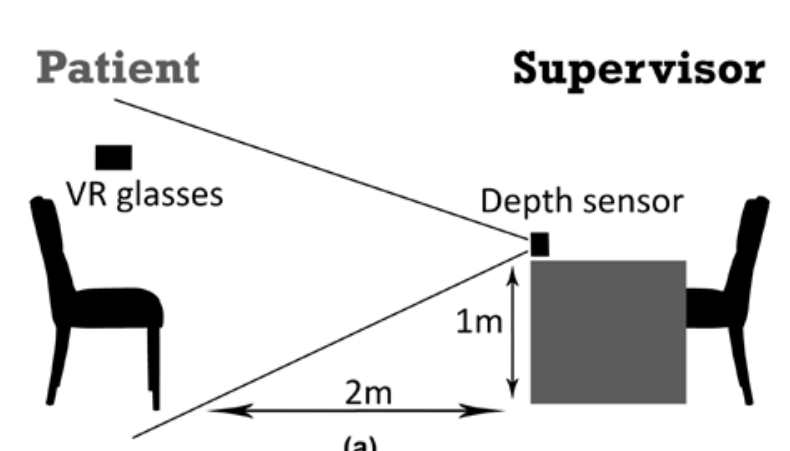

(a)

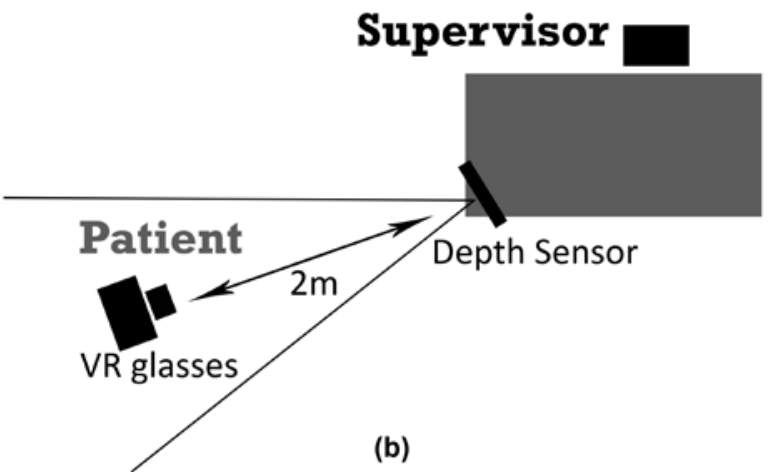

(b)

Figure 3. (a) Top down view showing the position of the devices, and the location of the patient and the supervisor. Around the patient should be space to avoid collision with any real objects. (b) Side view showing the position of the devices, and the location of the patient and the supervisor. The depth sensor should be located at approximately 1 meter above the floor. The patient has to be approximately 2 meters away from the depth sensor in order to be in the view range.

The interaction with the virtual environment is done through the VR glasses and the depth sensor. Some of the tests are visual or audio based, therefore the interaction is verbal; the supervisor collects the information and then they have to transcribe the results to a form. Other tests require the selection amongst different options. In these cases, a scope appears in the middle of the screen and using head movements the subject can select amongst the options. The results from these tests are automatically stored in a file.

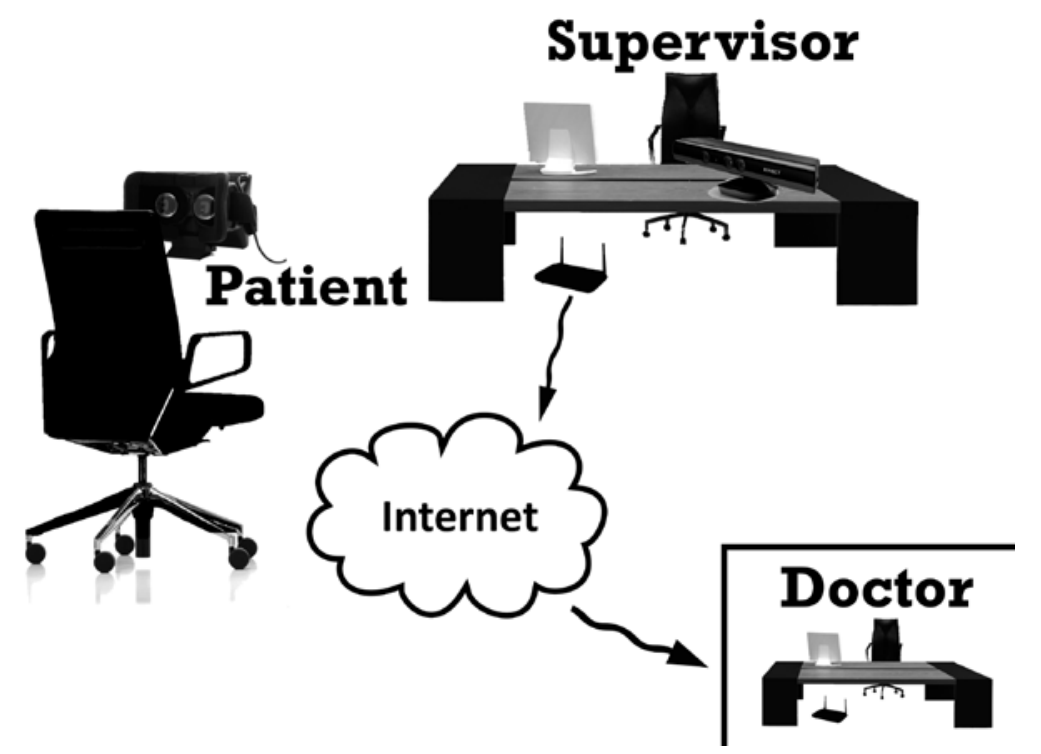

Figure 4. Early Detection Test setup. The patient is sat in front of the depth sensor, wearing the VR glasses. The supervisor (or the doctor) monitors the test from the computer or remotely as an e-health application. 
Once the patient is immersed on the VE, some general questions, such as gender and educational level, are displayed before starting the tests. The subject has to select the options using the scope. The subjects' avatar gender and the difficulty of the tests will change according to their selections.

\subsection{Virtual Objects Memorization test (VOM)}

This test is formed by memory tasks and it is split into three steps. The first step of the memory test is the recognition of six different virtual objects. The selected items are common everyday objects making it easier to identify and recognize them. The objective of this first task is to check if the patient has problems in recognizing quotidian objects (see Figure 5a). Once all the objects are recognized, a virtual monitor displays all of them in specific positions (the positions of these objects are the ones where they normally are located on a desk), see Figure 5b. Therefore, the patient is asked to memorize the objects but nothing is mentioned about their position.

During the second step, the subject is asked about the objects located at specific positions, in order to assess the visual and the associative memory of the patient. In more details, the desk is shown in front of the patient and a number appears in the position where an object was located and a list of the six possible objects is provided (see Figure 5c). The subjects are asked about the object that was located in the position indicated by the number and they have to select the name of the correct one. The fact that the objects were located in logical positions should help the user to select the right answers.

Finally, in the last task, interacting objects are placed in the virtual room and have to be located and memorized by the patient. Then, one of the objects is shown and the subject has to recall its interacting pair and their functionality. Once the third stage is completed, there is an extra task associated with the first step, where the patient is asked to recall the objects that were shown. The aim of the last two tasks is to evaluate the patient's short-term memory incorporating interacting objects and their functionalities. So, the novel part here is focused mainly on the combination of interacting objects with their functionalities.

Also, it should be mentioned that in order to proceed through the test, the subject has to select amongst different options. This selection is performed by aiming towards the desired option using the direction of view (scope), as we can see in figure 5 . Therefore, the patients have to move slightly their heads to fulfil the tasks.

The general aim of this test is to analyse patients' learning and memory cognitive domain (American Psychiatric Association, 2013). This test provides in total 21 points, if all tasks are completed successfully. Each point is associated to the recognition of the objects, the recall of their position and the recall of the objects themselves and their functionality. 


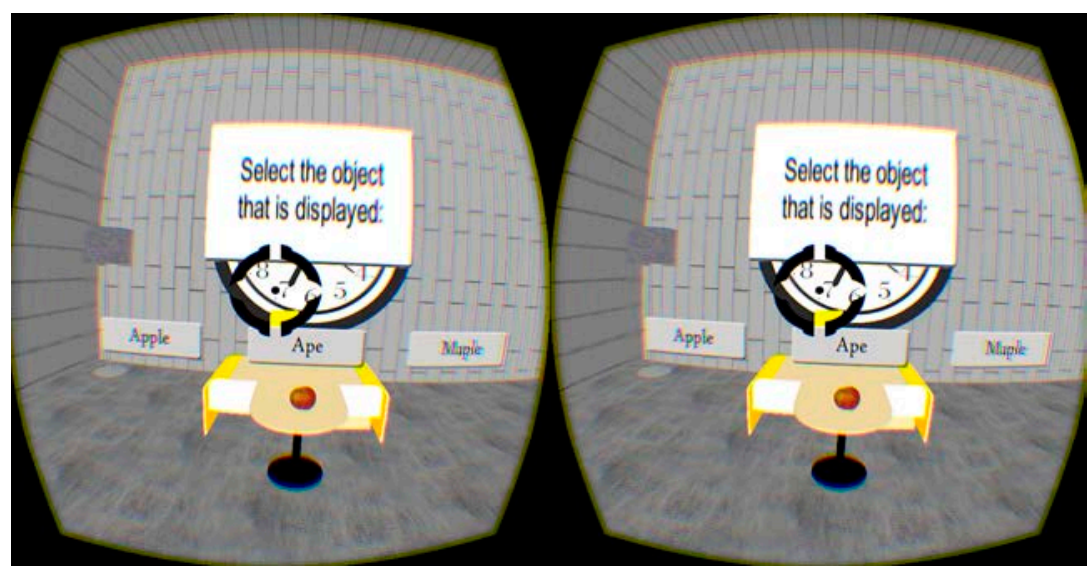

(a)

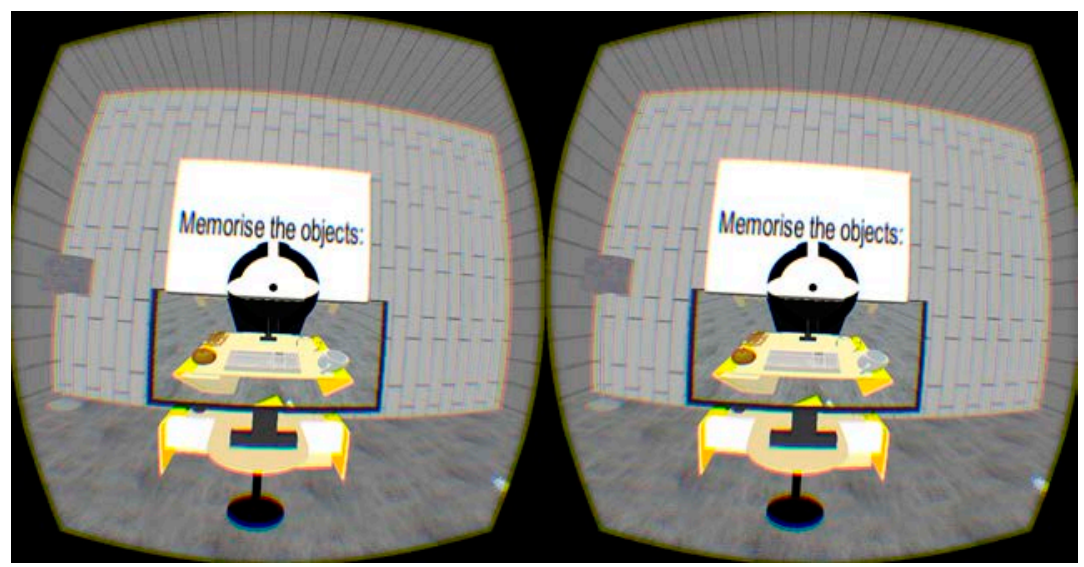

(b)

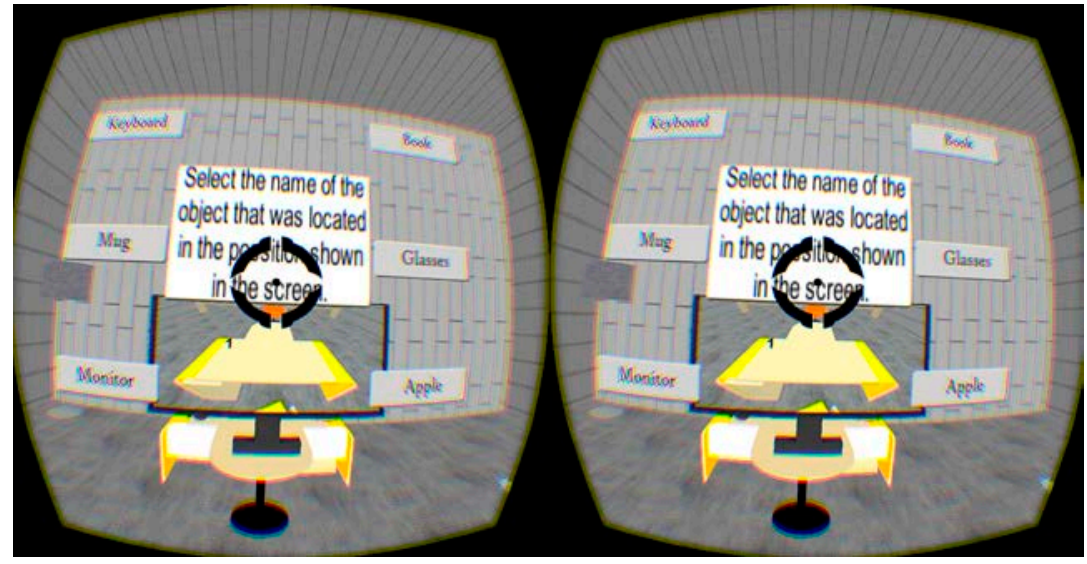

(c)

Figure 5. (a) Virtual Object Memorization tasks. Objects Recognition task where the patient has to identify and select the correct name of the object over the table. Virtual Object Memorization tasks. (b) The objects in their respective location is shown in order the patient memorises the objects. (c) Objects location task where the patient has to select the name of the object that was shown on the screen 


\subsection{Abnormal Objects Recognition test (AOR)}

The basis of this test is to evaluate if the patient is able to discern if something is abnormal or not within the virtual environment. This test is divided into three separate stages. During the first stage, the patient is asked to detect any abnormalities present in the room in order to analyse their perceptual-motor cognitive domain (American Psychiatric Association, 2013). Table 1 describes the abnormalities that can be found in the room. During the second stage, the subject is requested to look the clock that is located on one of the walls in the room. Shortly afterwards the patient is asked if the illumination (i.e. ambient light) of the room is in accordance with the time.

Table 1. Abnormalities in the room ordered by discovery difficulty. The potted plant and the car discovery difficulty is similar.

\begin{tabular}{|l|l|}
\hline Object & Abnormality \\
\hline Chair & Wanders around the room \\
\hline Mug & Spins \\
\hline \multirow{2}{*}{ Car } & Goes through the wall \\
\cline { 2 - 2 } & Change shape when is passing by the mirror \\
\hline Potted plant & Upside down \\
\hline Mirror & Does not reflect \\
\hline
\end{tabular}

Finally, the objective of the third stage is to evaluate the coordination of the patient when the physics of a mirror reflection are altered. An avatar, whose movements are linked with the patients' movements, is displayed in front of them. Therefore, it will look like a mirror; however, the movements of the avatar are reversed. Once the patients detect how the avatar moves, they are asked to perform specific movements with the avatar (see table 2). This last task allows the study of perceptual-motor and executive function impairment (American Psychiatric Association, 2013).

Table 2. Movements that the patient has to perform to move the avatar and their evaluation.

\begin{tabular}{|l|l|}
\hline Task & Answer and Evaluation \\
\hline $\begin{array}{l}\text { Raise your right hand. Is the avatar behaving as a } \\
\text { mirror? }\end{array}$ & No = 1 point \\
\hline $\begin{array}{l}\text { Bounce the body of the avatar towards the mug (there } \\
\text { is a mug on the right of the avatar) }\end{array}$ & First attempt $=1$ point \\
\hline Grab the mug & First attempt $=1$ point \\
\hline
\end{tabular}

This test can provide a maximum of 10 points. Seven points are associated with the correct detection of the abnormalities and three are associated with the correct movement of the avatar. 


\subsection{Virtual vs Real Sounds test (VRS)}

The main objective of this test is to detect if the patient is able to discern between real and virtual sounds in order to analyse their mental and cognitive flexibility (American Psychiatric Association, 2013). Different sounds are played during the test, such as the clock ticking, the rain, etc. (see table 3). The patient has to recognise and name all the sounds that they listen during the test. This part of the test tries to identify if the patient is still able to discern events through sounds. In addition, the virtual environment will provide some visual clues to help them with the identification (see Figure 6). Once the subjects have identified all the sounds, they are asked about the origin of the sounds. During this stage, the source of some sounds is coming either from the virtual environment and the real world reproduced by the supervisor. This second part requires the patient to recall all the sounds that were played and to identify which ones were reproduced by the supervisor.

This test gives a maximum score of 6 points. One point is obtained for each object that is recognized and categorized properly as real or virtual.

Table 3. Sounds played during VRS test

\begin{tabular}{|l|c|}
\hline Sound & Origen \\
\hline Ticking clock sound & Virtual \\
\hline Fly buzzing sound & Virtual \\
\hline Breaking glass sound & Virtual \\
\hline Closing door sound & Virtual \\
\hline Rain sound & Virtual \\
\hline Mobile phone & Real \\
\hline
\end{tabular}

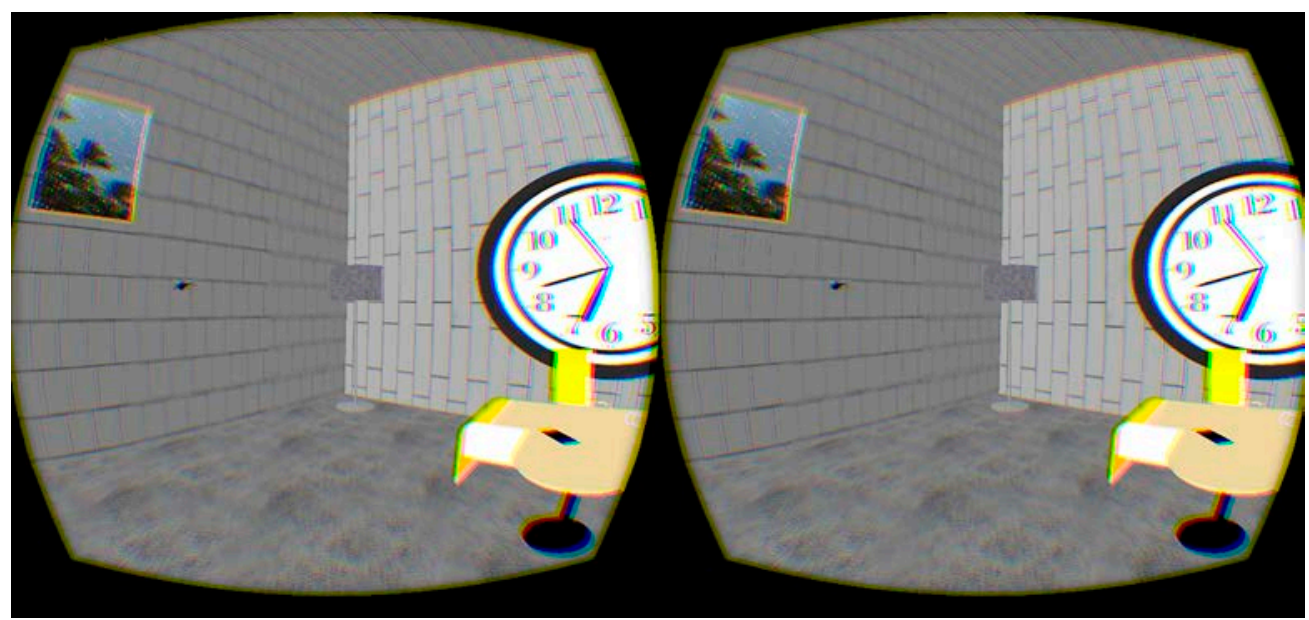

Figure 1. Virtual vs Real Sounds test. The image shows 4 sound sources/clues of the 6 used (Clock, fly, rain and mobile phone). The mobile phone sound is reproduced by the test supervisor 


\subsection{Adaptability to high IQ participants}

In order to reduce the effect of the subjects' educational level on the results, the difficulty of the test has been increased for participants with high educational level. The number of objects during the VOM test is increased; the high educated participants should recognize and recall 3 additional objects. During the AOR test, the mirror reflection abnormality detection is only required for high IQ participants and a new abnormality with a calendar showing an incorrect date is added. Finally, during the VRS test, when the mobile phone sound is played on the real world, a mobile phone in the virtual world will start moving trying to trick the high IQ subjects.

\section{RESULTS}

\subsection{Participants}

The 20 participants of this test are aged between 23 and 82 year old including both healthy people and Alzheimer's patients diagnosed with mild dementia less than a year ago. Furthermore, Alzheimer's patients in advanced conditions were tested but, the process was not possible to be completed. Half the subjects were male and the other half female of whom eleven had college or higher educational background and nine did not reach high school. It has to be mentioned that one of the patients was illiterate so the test was performed orally. Previously to the start of the test, it was confirmed that the subjects did not have any visual or auditory problem that could be a handicap while performing the tests.

\subsection{Data}

The main data collected during the experiment are the individual scores for each test. As it was explained in section 3, each test has a maximum score and each task has associated to a different number of points. In more details, regarding the recognition and memory tasks each recalled and recognized object scores 1 point.

In addition, patients have been also tested using other well-known state of the art Alzheimer screening tests in order to perform a comparative study and allow the evaluation of the proposed novel non-invasive diagnosis screening tests. All the tests were computerized so the patients have to use the computer through the whole process retaining also the same conditions among all tests. The screening tests used in our comparative study are Dr. Oz Memory Quiz (test based on St. Louis University Mental Status Examination (SLUMS)) (Cruz-Oliver, Malmstrom, Roegner, Tumosa \& Grossberg, 2014); Visual Association Test (Lindeboom, Schmand, Tulner, Walstra \& Jonker, 2002); and Dichotic Listening Test (DLT) (Duchek \& Balota, 2005). Two different results were collected from the DLT: DLTre accounts the predominance of the right ear when recalling digits and DLT accounts the number of digits recalled. 


\subsection{Experiments and outcomes}

The mean results for each test in relation to the subject's health status are shown in Table 4. It is observed that healthy patients have achieved results close to the maximum score of each test and the Alzheimer's patients have obtained significantly lower scores in comparison with the health participants. For instance, healthy subjects have obtained a mean of 0.924821 whereas for Alzheimer's case the score was 0.238100 in the Virtual Objects Memorization test. This separation between results is also apparent in the other state of the art tests, such as the Dichotic Listening test (DLTre) with results in the following ranges Healthy=0.986842 and Alzheimer 0.375000 .

Table 5 demonstrates that the $p$-value of the novel tests proves that their results are correlated with the healthy and Alzheimer status of the patients. When it comes to sensitivity and specificity in relation to the detection of healthy patients, the results are perfect. This suggests that more subjects need to be tested in order to corroborate the results of the novel Alzheimer screening tests.

Table 4. Mean, number of cases of the tests regarding the Healthy and Alzheimer patients

\begin{tabular}{|l|l|l|l|l|l|l|l|l|}
\hline & Age & DrOz & VAT & DLT & DLTre & VOM & AOR & VRS \\
\hline Healthy & 42.47 & .900953 & .894732 & .889253 & .986842 & .924821 & .826316 & .833311 \\
\hline Alzheimer & 78.00 & .529400 & .000000 & .437500 & .375000 & .238100 & .200000 & .333300 \\
\hline
\end{tabular}

Table 5 also demonstrates that most of the results of the test are not correlated with the age and the educational level of the patients, since the p-values do not reject the Null hypothesis. Regarding the novel AOR test, it results $p$-values of 0.003 (age) and 0.004 (educational level), indicating a relationship with those factors. Therefore, tasks that are more difficult are available for patients with high educational background in order to eliminate this correlation. When it comes to the relation between the results and the gender of the participants, the results of the male and female participants are not correlated (see table 5).

\subsection{Qualitative evaluation of the proposed system}

At the end of the test, patients were asked to evaluate the application. They had to fill a form evaluating the quality of the application in terms of interaction; the simplicity of the instructions/process and the comfort of the glasses. Additionally, the patients had the option to provide any additional comments about the application.

The average evaluation of the application was positive. Some of the subjects commented that the glasses were not comfortable at the end of the test and that the graphics could be improved. Nevertheless, most of the participants evaluated the application as motivating and interesting; and the interaction and instructions as simple and easy to understand. 
Table 5. T-test for each test according to the healthy and Alzheimer's cases. The p-value (Sig. (2tailed)) is less than 0.01 so the Null Hypothesis is rejected

\begin{tabular}{|c|c|c|c|c|c|}
\hline & \multicolumn{4}{|c|}{ t-test for Equality of Means } \\
\hline & & Sig. (2-tailed) & Sig. (2-tailed) & Sig. (2-tailed) & Sig. (2-tailed) \\
\hline & & Dementia & Age & Educational Level & Gender \\
\hline \multirow[t]{2}{*}{ DrOz } & e.v.a ${ }^{\mathrm{a}}$ & .000 & .033 & .067 & 1.000 \\
\hline & e.v.n.a ${ }^{b}$ & & .088 & .104 & 1.000 \\
\hline \multirow[t]{2}{*}{ VAT } & e.v.a & .001 & 325 & .462 & 615 \\
\hline & e.v.n.a & & 395 & .492 & .615 \\
\hline \multirow[t]{2}{*}{ DLT } & e.v.a & .000 & .038 & .111 & 616 \\
\hline & e.v.n.a & & .076 & .142 & 619 \\
\hline \multirow[t]{2}{*}{ DLTre } & e.v.a & .000 & .198 & 262 & .400 \\
\hline & e.v.n.a & & 314 & 326 & 410 \\
\hline \multirow[t]{2}{*}{ VOM } & e.v.a & .000 & .052 & .045 & .908 \\
\hline & e.v.n.a & & .128 & .084 & .909 \\
\hline \multirow[t]{2}{*}{ AOR } & e.v.a & .000 & .003 & .004 & .433 \\
\hline & e.v.n.a & & .020 & .013 & .435 \\
\hline \multirow[t]{2}{*}{ VRS } & e.v.a & .000 & .061 & .078 & .818 \\
\hline & e.v.n.a & & .113 & .104 & .818 \\
\hline
\end{tabular}

b Equal Variances Not Assumed

Table 6 presents the overall evaluation of the tool performed by the participants. The interaction with the application (keyboard, mouse, depth sensor and virtual reality glasses), the quality of the instructions (written and/or oral provided by the supervisor) and the comfort of the VR glasses was evaluation from 0 points (really bad) to 5 points (really good). The evaluation obtained is positive, since the average result of all the categories is above 3 points, with elder people being more enthusiastic with new technologies probably due to the completely new to them features and capabilities.

Table 6. Tool evaluation by the participants. Four characteristics were evaluated from 0 (Very bad) to 5 (Very good)

\begin{tabular}{|c|c|c|c|c|}
\hline TYPE & Mouse Inter & Kinect Inter & Instructions & VR Glasses \\
\hline$>=\mathbf{5 0}$ & $4.17(0.983)$ & $4.00(1.155)$ & $4.43(0.787)$ & $3.86(0.9)$ \\
\hline$<\mathbf{5 0}$ & $4.33(0.778)$ & $3.33(0.985)$ & $4.17(0.835)$ & $3.50(1.0)$ \\
\hline
\end{tabular}

\section{CONCLUSIONS}

Alzheimer is a disease without foreseen cure, that is affecting more people every year and whose research and associated services (care) cost is rapidly increasing. Our objective is to provide affordable tools that would help to improve the current detection systems. 
It was possible to design, develop and test a novel low cost, non-invasive, cognitive Alzheimer screening tool for diagnosis based on VEs. VEs have started to form part of medical treatments and diagnosis methods. They provide secure and controlled environments and increase the effectivity of the tests. These facts have further been proved with our application since the results show that it is possible to create an accurate Alzheimer's diagnostic screening system. Novel methods for detection of Alzheimer's disease based on VEs were introduced. The proposed tests are focused on the evaluation of memory loss related to common objects, recent events, the ability to recognise abnormalities and, to differentiate between virtual worlds and reality. The proposed approaches were evaluated in a comparative study with well-known state of the art cognitive tests. Finally, the overall evaluation indicates similar outcomes with the classic state of the art cognitive tests, while the adaptability of the proposed methodology reduces the ceiling effect in the case of high IQ patients.

\section{REFERENCES}

[1] Alzheimer's Association (2016). Alzheimer's \& Dementia. Available from: $<$ http://www.alz.org/>. [11 March 2016]

[2] Whalley L.J. \& Breitner J. C. S. (2009). Fast Facts: Dementia. Health Press.

[3] Alzheimer's Research UK (2012). Alzheimer's and dementia: your questions answered. Alzheimer's Research UK

[4] Tarnanas I., Tsolaki M., Nef T., M Müri R. \& Mosimann U. P. (2014). "Can a novel computerized cognitive screening test provide additional information for early detection of Alzheimer's disease?" Alzheimer's \& Dementia.

[5] Wimo A., \& Prince M. J. (2010). World Alzheimer Report 2010: the global economic impact of dementia. Alzheimer's Disease International.

[6] Lewis T., Synowiec C., Lagomarsino G. \& Schweitzer J. (2012). E-health in low-and middleincome countries: findings from the Center for Health Market Innovations. Bulletin of the World Health Organization, 90(5), 332-340.

[7] López-de-Ipiña K., Alonso J. B., Barroso N., Faundez-Zanuy M., Ecay M., Solé-Casals J., Travieso C. M., Estanga A. \& Ezeiza A. (2012). New approaches for Alzheimer's disease diagnosis based on automatic spontaneous speech analysis and emotional temperature. In Ambient Assisted Living and Home Care (pp. 407-414). Springer Berlin Heidelberg.

[8] Rowe C. \& Healy S. D. (2014). Measuring variation in cognition. Behavioral Ecology, 25(6), 1287-1292.

[9] Wild K., Howieson D., Webbe F., Seelye A. \& Kaye J. (2008). Status of computerized cognitive testing in aging: a systematic review. Alzheimer's \& Dementia, 4(6), 428-437.

[10] Taekman J. M. \& Shelley K. (2010). Virtual environments in healthcare: immersion, disruption, and flow. International anesthesiology clinics, 48(3), pp: 101-121.

[11] Tarnanas I., Schlee W., Tsolaki M., Müri R., Mosimann U., \& Nef T. (2013). Ecological validity of virtual reality daily living activities screening for early dementia: longitudinal study. JMIR Serious Games, 1(1), e1. 
[12] Weibel D. \& Wissmath B. (2011). "Immersion in Computer Games: The Role of Spatial Presence and Flow,” International Journal of Computer Games Technology, vol. 2011, Article ID 282345, 14 pages, 2011. doi:10.1155/2011/282345

[13] Abe Y., Toya M. \& Inoue M. (2013). Early detection system of senile dementia by behavior sensing. Consumer Electronics (ISCE), 2013 IEEE 17th International Symposium. pp: 67-68

[14] Han S. D., Gruhl J., Beckett L., Dodge H. H., Stricker N. H., Farias, S. \& Mungas D. (2012). Beta amyloid, tau, neuroimaging, and cognition: sequence modeling of biomarkers for Alzheimer's Disease. Brain imaging and behavior,6(4), 610-620.

[15] Kadmiri, N. El. (2015). Biomarkers of AD Biomarkers of Alzheimer 's Disease, (MARCH), 11-14.

[16] Unay D. \& Ekin A. (2011).Dementia diagnosis using similar and dissimilar retrieval items. In Biomedical Imaging: From Nano to Macro. pp: 1889-1892

[17] Akgul C. B. \& Ekin A. (2010). A Probabilistic Information Fusion Approach to MR-based Automated Diagnosis of Dementia. In Pattern Recognition (ICPR).pp: 265-268

[18] Aztiria A., Ugarte M. \& Izaguirre A. (2013). Gait Analysis for Identifying Parameters Related to Dementia in Intelligent Environments. Intelligent Environments (IE), pp:3-6.

[19] Fountoulakis K. N., Tsolaki M., Chantzi H. \& Kazis, A. (2000). Mini mental state examination (MMSE): a validation study in Greece. American Journal of Alzheimer's Disease and Other Dementias, 15(6), pp: 342-345.

[20] Cruz-Oliver D. M., Malmstrom T. K., Roegner M., Tumosa N. \& Grossberg G. T. (2014). Cognitive deficit reversal as shown by changes in the Veterans Affairs Saint Louis University Mental Status (SLUMS) examination scores 7.5 years later. Journal of the American Medical Directors Association, 15(9), 687-e5.

[21] Lindeboom J., Schmand B., Tulner L., Walstra G., \& Jonker C. (2002). Visual association test to detect early dementia of the Alzheimer type. Journal of Neurology, Neurosurgery \&Psychiatry 73(2) pp: 126-133

[22] Duchek J. M. \& Balota D. A. (2005). Failure to control prepotent pathways in early stage dementia of the Alzheimer's type: evidence from dichotic listening. Neuropsychology, 19(5), pp: 687.

[23] Oppy G. \& Dowe D. (2011). The turing test. The Stanford Encyclopedia of Philosophy.

[24] Quental N. B. M., Brucki S. M. D. \& Bueno O. F. A. (2013). Visuospatial Function in Early Alzheimer's Disease-The Use of the Visual Object and Space Perception (VOSP) Battery. PLOS ONE, 8(7).

[25] Pereira M. L. F., Marina von Zuben A. C., Aprahamian I. \& Forlenza O. V. (2014). Eye movement analysis and cognitive processing: detecting indicators of conversion to Alzheimer's disease. Neuropsychiatric disease and treatment,10, 1273.

[26] Cho, S., Ku, J., Cho, Y. K., Kim, I. Y., Kang, Y. J., Jang, D. P., \& Kim, S. I. (2014). Development of virtual reality proprioceptive rehabilitation system for stroke patients. Computer Methods and Programs in Biomedicine, 113(1), 258-265.

[27] Crutcher M. D., Calhoun-Haney R., Manzanares C. M., Lah J. J., Levey A. I. \& Zola S. M. (2009). Eye tracking during a visual paired comparison task as a predictor of early dementia. American journal of Alzheimer's disease and other dementias. 
[28] American Psychiatric Association. (2013). Diagnostic and Statistical Manual of Mental Disorders (DSM-5®). American Psychiatric Pub.

[29]Juan Manuel Fernández Montenegro and Vasileios Argyriou, Diagnosis of Alzheimer's disease based on Virtual Environments, IEEE IISA2015, Corfu 2015 